\title{
Research on Algorithm to Improve Accuracy of Temperature Measurement of Moving Strong Reflector
}

\author{
Shaoliang Wei $(\mathbb{D}$, Yuanjia Yang $(\mathbb{D}$, and Fengyu Cheng $(\mathbb{D}$ \\ School of Mechanical and Power Engineering, Henan Polytechnic University, Jiaozuo 454003, China \\ Correspondence should be addressed to Shaoliang Wei; wsl_ify@163.com
}

Received 19 April 2021; Accepted 13 September 2021; Published 4 October 2021

Academic Editor: Carlos Marques

Copyright ( 2021 Shaoliang Wei et al. This is an open access article distributed under the Creative Commons Attribution License, which permits unrestricted use, distribution, and reproduction in any medium, provided the original work is properly cited.

\begin{abstract}
The roll is necessary and important equipment in aluminum processing, and its surface temperature will change its thermal expansion. The gap shape between rolls will change accordingly, affecting the quality of aluminum products. Therefore, it is important to monitor and control the roll surface temperature. The roller is a highly reflective body that rotates continuously during work. This article proposes an infrared temperature sensor to measure its surface temperature. Through field investigation and a literature review, we know that a roller's motion will affect the accuracy of its surface temperature measurement. An infrared temperature measurement compensation algorithm based on rotation speed is constructed to eliminate this influence. Experimental results show that this method can make up for the temperature measurement error caused by the change of rotation speed and hence improve measurement accuracy. The algorithm is simple and adaptable and provides a new method to improve the accuracy of temperature measurement given speed change.
\end{abstract}

\section{Introduction}

With the development of electronic science and technology, the traditional contact temperature measurement method has been unable to meet the needs of some fields, and the demand for noncontact, long-distance temperaturemeasurement technology is growing. Traditional temperature measurement requires contact with the subject or object, which can be inconvenient. In industrial temperature measurement, it takes a certain time for the measurement element and the object to reach thermal equilibrium, which cannot realize real-time monitoring and places high requirements on the measuring element, which is prone to damage. Noncontact temperature measurement has no special requirements for the heat-resistant performance of the sensor, which avoids mutual interference between the sensor and the object. Moreover, the sensor has a large temperature measurement range, no thermal inertia, and fast response speed, which frequently meet the requirements for the accuracy and range of temperature measurement. As a typical noncontact technology, infrared temperature measurement has the advantages of high accuracy, a wide temperature measurement range, real-time observation, and automatic control [1-3].

During the strip rolling process, the heat of the rolled product and that released by its deformation will increase the temperature of the roll and generate thermal convexity along its axial direction. This not only affects the strip quality; the roll surface is repeatedly exposed to rapid heating and cooling; hence, it is prone to fatigue damage and production accidents $[4,5]$. To ensure safety in production and improve the quality of rolled materials, it is essential to control the temperature of the rolls and set optimal cooling conditions; the temperature of the roll will rise or drop during the rolling process, which requires monitoring of the temperature distribution on the roll surface in real time. Researchers currently focus on the use of infrared temperature sensors to measure the temperature of stationary object surfaces and analyze the impact of object surface emissivity, measurement distance, and environmental factors on temperature measurement accuracy. For moving object surfaces, the literature on real-time temperature measurement and control is scarce, and there is little research on the influence of speed condition on the accuracy of infrared temperature 


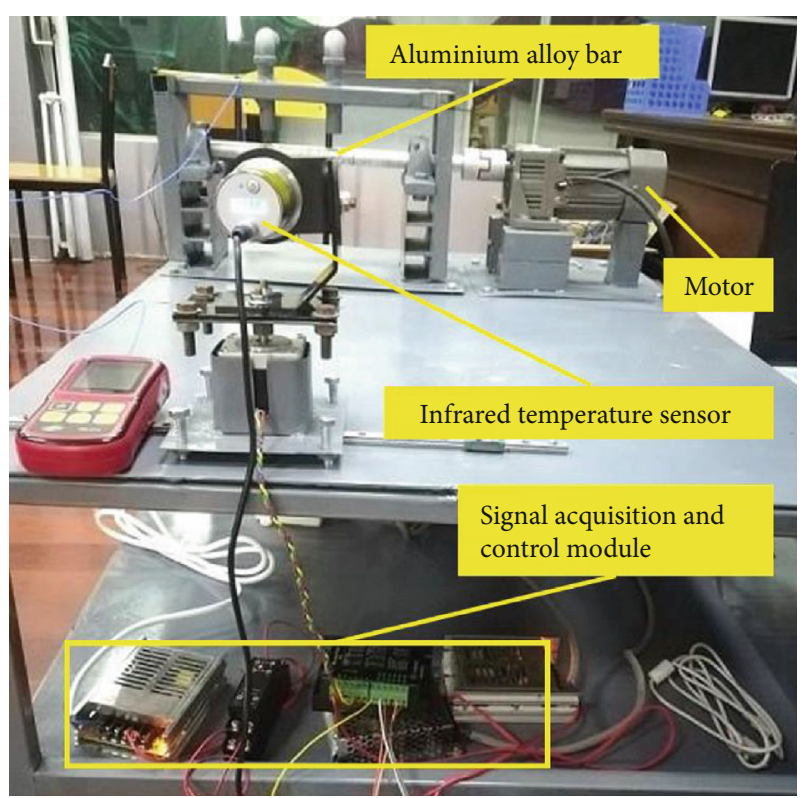

Figure 1: Temperature measuring device.

TABLE 1: Infrared temperature measurement results when the rotation speed is zero.

\begin{tabular}{lccc}
\hline Group & $\begin{array}{c}\text { Actual } \\
\text { temperature }\left({ }^{\circ} \mathrm{C}\right)\end{array}$ & $\begin{array}{c}\text { Infrared temperature } \\
\text { measurement }\left({ }^{\circ} \mathrm{C}\right)\end{array}$ & $\begin{array}{c}\text { Error } \\
\left({ }^{\circ} \mathrm{C}\right)\end{array}$ \\
\hline 1 & 181.7 & 182.7 & 1 \\
2 & 175.6 & 176.4 & 0.8 \\
3 & 170.4 & 171.3 & 0.9 \\
4 & 166.4 & 166.4 & 0 \\
5 & 161.8 & 162.9 & 1.1 \\
6 & 156.1 & 157.1 & 1 \\
7 & 151.4 & 152.3 & 0.9 \\
8 & 147.3 & 148.3 & 1 \\
9 & 142.9 & 142.9 & 0 \\
10 & 138.2 & 139.4 & 1.2 \\
11 & 134.1 & 135.2 & 1.1 \\
12 & 130.7 & 131.6 & 0.9 \\
13 & 127.4 & 127.4 & 0 \\
14 & 122.4 & 123.1 & 0.7 \\
15 & 117.6 & 118.5 & 0.9 \\
16 & 114.8 & 115.3 & 0.5 \\
17 & 110.4 & 111.4 & 1 \\
18 & 107.3 & 108.3 & 1 \\
\hline
\end{tabular}

measurement. Based on the theory of infrared temperature measurement, this experiment reveals that the change of the rotation speed of the roll will affect the accuracy of the surface temperature measurement of the strong reflector. An infrared temperature compensation algorithm based on the speed of the strong reflector is established so as to improve the temperature measurement accuracy of its surface.

\section{The Principle of Infrared Temperature Measurement}

In nature, charged particles inside any substance are in a state of constant motion. When the temperature of an object is higher than the thermodynamic temperature of $0 \mathrm{~K}$, or $-273.15^{\circ} \mathrm{C}$, the object will continuously radiate infrared energy [6]. The amount of infrared energy radiated by an object according to the wavelength distribution is closely related to its surface temperature. The precise surface temperature can be measured, by measuring the infrared energy radiated by the object.

The theory of blackbody radiation states that a radiating object can be considered as a black body under ideal conditions. It absorbs energy of any wavelength without reflecting and transmitting energy. All the energy it absorbs will be radiated outward. As shown in equation (1), Planck's law gives the relationship between the radiative intensity of a black body and its temperature and wavelength $[7,8]$.

$$
M_{b b}(T, \lambda)=\frac{C_{1}}{\lambda^{5}} \times \frac{1}{e^{C_{2} / \lambda T}-1},
$$

where $M_{b b}(T, \lambda)$ is the radiation energy density of blackbody (unit: $\mathrm{W} /\left(\mu \mathrm{m} \cdot \mathrm{m}^{2}\right)$ ); $C_{1}$ is the first radiation constant $\left(C_{1}=2 \pi h c^{2}=(3.7415 \pm 0.0003) \times 10^{-16} \mathrm{~W} \cdot \mathrm{m}^{2}\right) ; C_{2}$ is the second radiation constant $\left(C_{2}=h c / k=(1.43879 \pm 0.00019) \times 10^{-2} \mathrm{~m} \cdot \mathrm{K}\right)$; and $\lambda$ is the radiation wavelength of light wave (unit: $\mu \mathrm{m}$ ).

At any temperature, an object that can completely absorb radiant energy of any wavelength projected onto its surface is called an absolute blackbody. Its absorption coefficient is equal to 1 , while the reflection coefficient is zero. Except for a cavity designed according to certain requirements, which is an approximate blackbody, an absolute blackbody is not available in nature. The surface reflection coefficient of all objects is less than 1 , and these are called gray bodies. In general, the infrared radiation of a gray body is not directly measured, but the ratio of the infrared radiation of a blackbody at the same temperature is used for denoting the characteristics of the actual measured object $[9,10]$. By comparing the relationship between the measured object and the normal radiance of a blackbody at various temperatures and wavelengths, the following relationship can be obtained.

$$
M=\varepsilon(T, \lambda) \cdot M_{b b}
$$

where $M$ is the actual radiation received and $\varepsilon$ is the emissivity, which is a function of temperature and wave length. The emissivity $\varepsilon$ of most thermal radiation objects changes slowly with the wavelength and has strong robustness. Therefore, the emissivity can be considered a constant in many cases.

According to the Stefan-Boltzmann law,

$$
M_{b b}=\sigma \cdot T^{4}
$$

where $\sigma$ is the Stefan-Boltzmann constant 


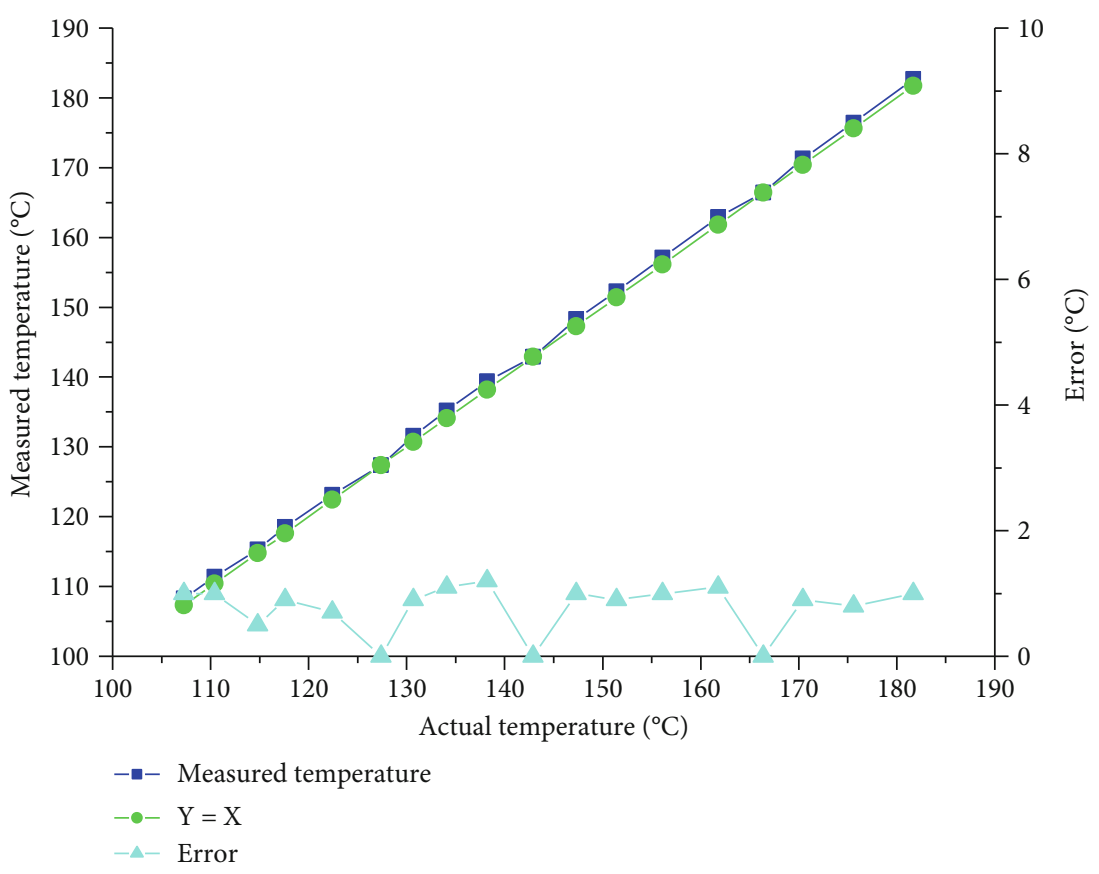

Figure 2: Comparison between the actual temperature and measured temperature when the rotation speed is zero.

$\left(\sigma=5.67032 \times 10^{-8}\left(\mathrm{~W} \cdot \mathrm{m}^{-2} \cdot \mathrm{K}^{-4}\right)\right)$ and $T$ is the thermodynamic temperature of the object.

This law indicates that the emissivity of a blackbody is proportional to the fourth power of the thermodynamic temperature. This holds not only for a blackbody but also for any real object. The measured radiance of a thermal radiation object is

$$
M=\varepsilon(T) \sigma T^{4}
$$

where $\varepsilon(T)$ represents the emissivity of materials in the full wavelength range at temperature $T$, also known as the blackness coefficient. Equation (4) provides a theoretical basis for the temperature measurement of a radiation thermometer.

Based on the theory of infrared temperature measurement, a measured object in the actual environment will radiate forms of energy other than its own infrared energy. It can be seen from the Stefan-Boltzmann law that the surface of any object continuously emits radiant energy except in the state of absolute zero. Under the condition that the external body does not supply any form of energy, its radiant energy is realized by consuming its internal energy. At the same time, the temperature of the object gradually decreases and continues to drop to absolute zero. However, this does not actually occur, because other objects around the radiating object are also radiating, and some of this will be absorbed by the object and converted to its internal energy. The radiation and absorption of energy by each object always occur simultaneously. When the temperature of an object is higher than that of the surrounding objects, its radiant energy exceeds the absorbed energy; hence, the object has a net energy loss. At this point, its temperature will decrease. When the temperature of an object is lower than that of objects around it, it will absorb more energy than it emits, so its temperature will increase. When an object and its surrounding environment are at the same temperature, the energy it emits is the same as it absorbs; i.e., it is in a state of radiation heat balance, and its temperature remains unchanged [11-14].

When the surface temperature of the object is measured, the energy radiated by the object does come not only from the object itself, also known as the radiation energy, but also from the ambient light irradiation temperature. This part of the energy will be received by the infrared temperature measurement system after being reflected on the object surface, which will result in inaccurate measurement $[15,16]$. Except for this, the infrared temperature measurement system will receive some energy from the measured object, which further influences the accuracy. The optical axis of the sample used in this experiment has a good transmission texture, so the transmitted energy can be ignored.

This article mainly discusses whether the infrared energy radiated from the sample surface received by the infrared temperature sensor will change significantly at different speeds, whether the change in the amount of radiation has a connection with the rotation speed of the measured object, and whether the error can be calibrated and reduced by a compensation equation. According to the principle of infrared temperature measurement, changes in radiant energy will ultimately be accounted for in changes in measurement temperature. This intuitive change is used for studying the effect of the optical axis speed on the accuracy of infrared temperature measurement.

\section{Experimental Test and Analysis of Results}

The roller in aluminum plate processing equipment has the characteristics of a smooth surface and strong reflection, and 

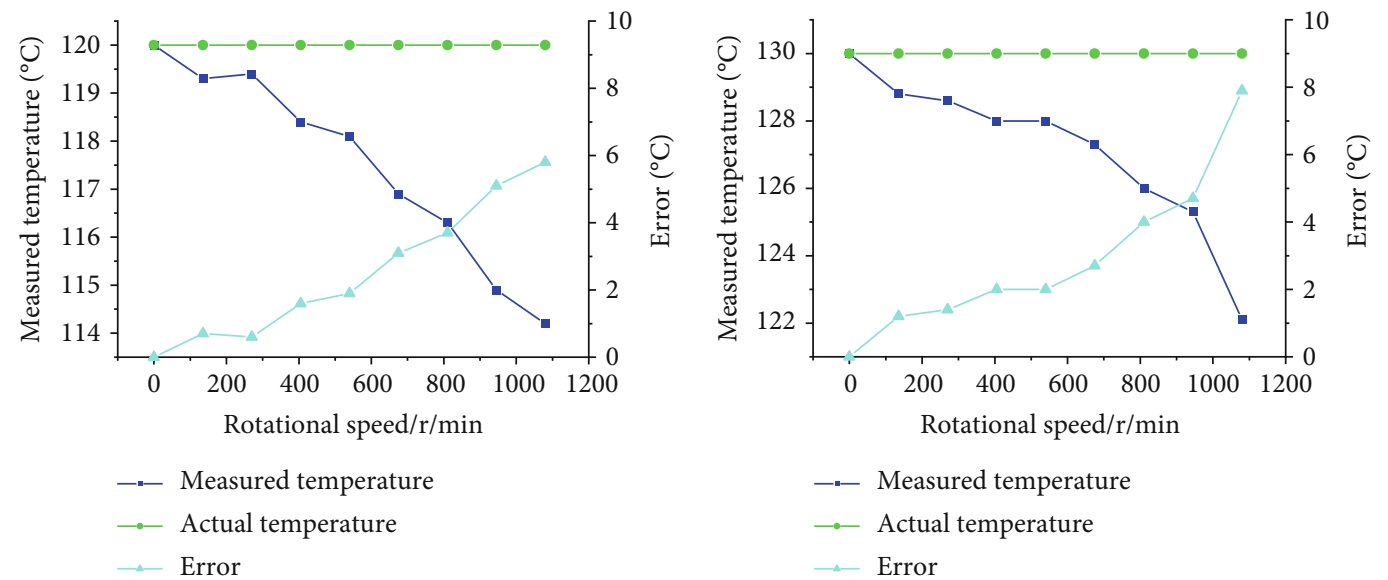

(a) $120^{\circ} \mathrm{C}$

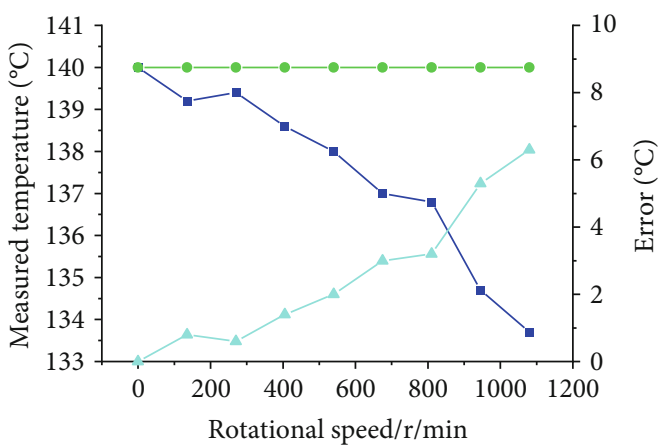

(b) $130^{\circ} \mathrm{C}$

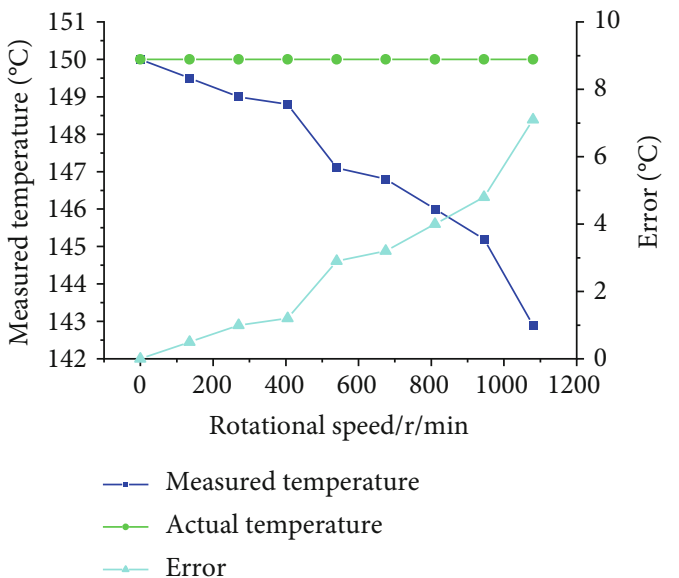

(c) $140^{\circ} \mathrm{C}$

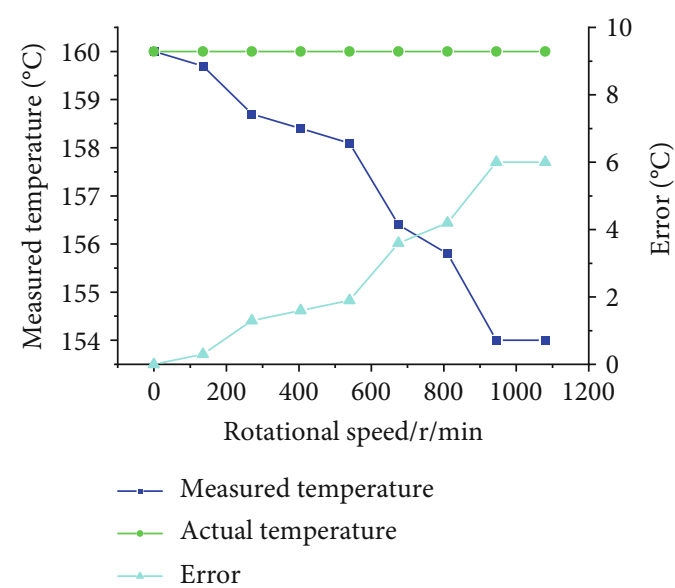

(d) $150^{\circ} \mathrm{C}$

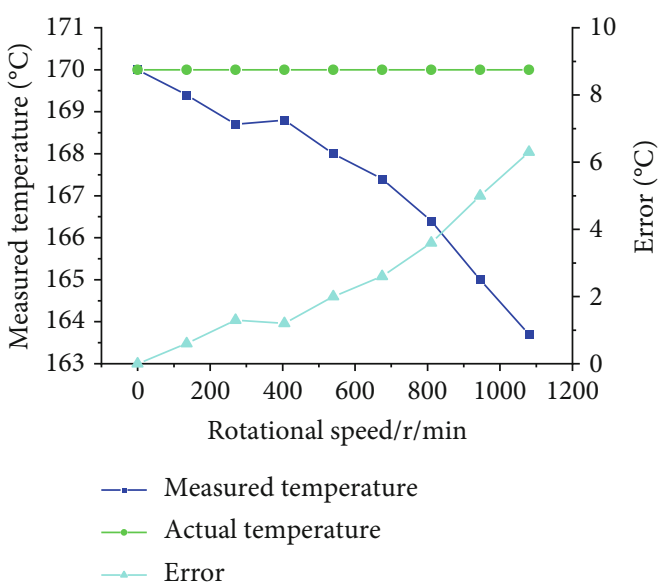

(e) $160^{\circ} \mathrm{C}$

(f) $170^{\circ} \mathrm{C}$

FIGURE 3: Measurement results and errors by the speed at different temperatures.

it is fine-grained, without transmission. In this test, an aluminum alloy rod with good reflective performance was selected as the test object. As shown in Figure 1, the alloy rod was on a bracket with a set height through a bearing on which it could rotate. The infrared temperature sensor was mounted on another bracket at a distance of $500 \mathrm{~mm}$ from the alloy rod. The emission layer of the infrared tem- perature sensor was located on the horizontal plane of the central plane of the measured object. An enclosed shading space was designed to reduce the impact of light in the environment and simplify the experiment. All experiments were completed in this space, whose temperature was kept constant to eliminate the impact of environmental changes on the accuracy of temperature measurement. 


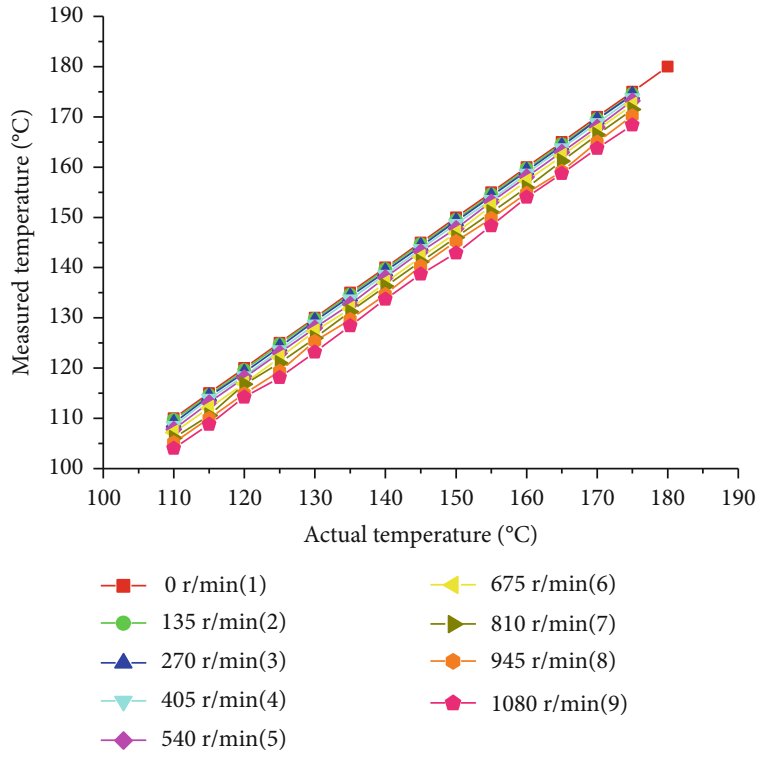

FIgURE 4: Comparison of actual and measured temperature at different speeds.

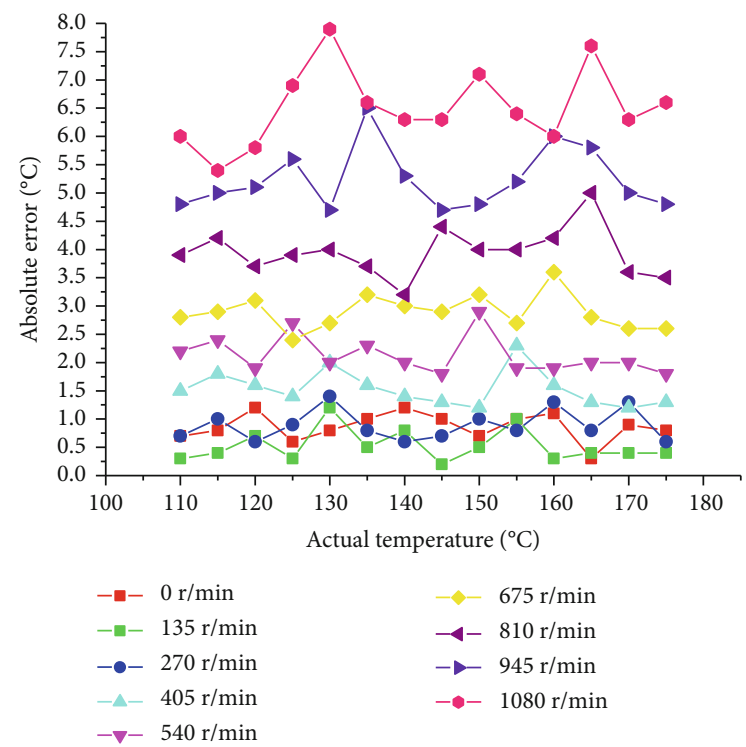

FIGURE 5: Absolute error of measured temperature at different speeds.

This article focuses on the influence of different speeds of the test object on temperature measurement accuracy. In the experiment, the normal static test experiment (rotation speed zero) and the test experiment under different speed conditions were carried out. By comparing the measured data, the results of infrared temperature measurement under different rotation speeds were analyzed to determine the influence of rotation speeds on the temperature measurement results.

3.1. Static Test in the Normal Direction (i.e., Rotation Speed Is Zero). In the experiment, an aluminum alloy bar with strong reflective characteristics was chosen as the measure-

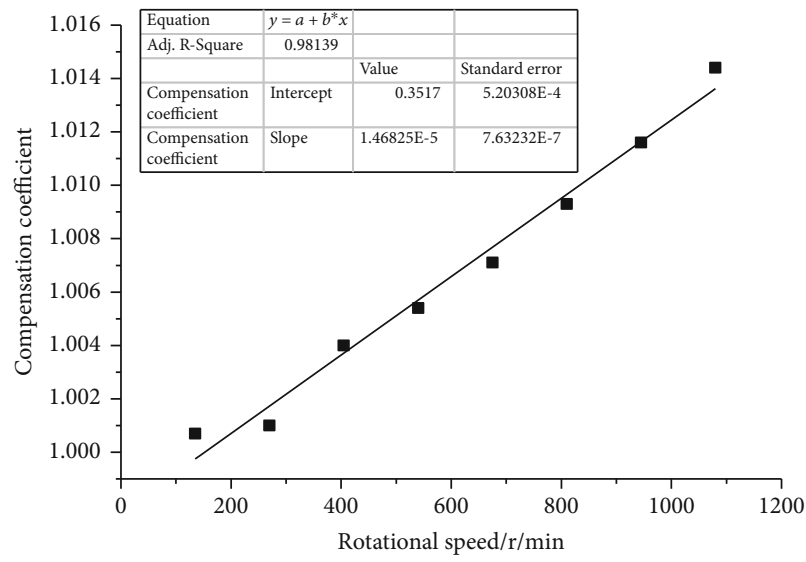

FIGURE 6: Relationship between compensation coefficient and rotation speed.

ment object. According to the IS laser targeting infrared sensor manual, the emissivity range of the aluminum alloy bar was $0.02-0.1$. The emittance of the measured object was determined to be 0.058 by repeated measurement of a fixed point, and the error was within $2^{\circ} \mathrm{C}$. The test results are shown in Table 1 and Figure 2.

In order to judge the measurement accuracy of infrared temperature sensor, the contact temperature sensor with higher accuracy is used for comparative experiment. In the experiment, the measured results of K-type thermocouple are taken as the actual temperature. In Figure 2, the $X$-axis and $Y$-axis represent the actual temperature and the temperature measured by the infrared sensor, respectively. Observing Figure 2, we can find that the measurement curve is relatively smooth without extreme values, which is basically consistent with the $Y=X$ curve (i.e., the actual temperature is equal to the measured temperature). Observing and analyzing the changes in the actual temperature and the temperature measured by the infrared temperature sensor in Table 1, it can be found that the difference between the measured and actual temperature value did not exceed $2^{\circ} \mathrm{C}$, and most were below $1^{\circ} \mathrm{C}$, which meets the requirements of temperature measurement error. The instability of absolute error is caused by the variation of light conditions and the different reaction time of thermocouple and infrared temperature sensor.

3.2. Temperature Measurement of Infrared Sensors at Different Speeds. The selected aluminum alloy bar can be determined by the above experiments, and its emissivity is 0.058. In the experiment, the emissivity was fixed, and the temperature of the measured object remained constant. The experiment is performed by changing the rotation speed of the aluminum alloy bar. Through the analysis of the measurement results, the influence of the speed condition on the temperature measurement of the strong reflector is further studied. The fixed temperatures of the bar selected in the experiment were $130^{\circ} \mathrm{C}, 140^{\circ} \mathrm{C}, 150^{\circ} \mathrm{C}, 160^{\circ} \mathrm{C}$, and $170^{\circ} \mathrm{C}$. The speed was $135 \mathrm{r} / \mathrm{min}, 270 \mathrm{r} / \mathrm{min}, 405 \mathrm{r} / \mathrm{min}, 540 \mathrm{r} / \mathrm{min}$, $675 \mathrm{r} / \mathrm{min}, 810 \mathrm{r} / \mathrm{min}, 945 \mathrm{r} / \mathrm{min}$, and $1080 \mathrm{r} / \mathrm{min}$. Changing the speed of the rolls to carry out different experiments 


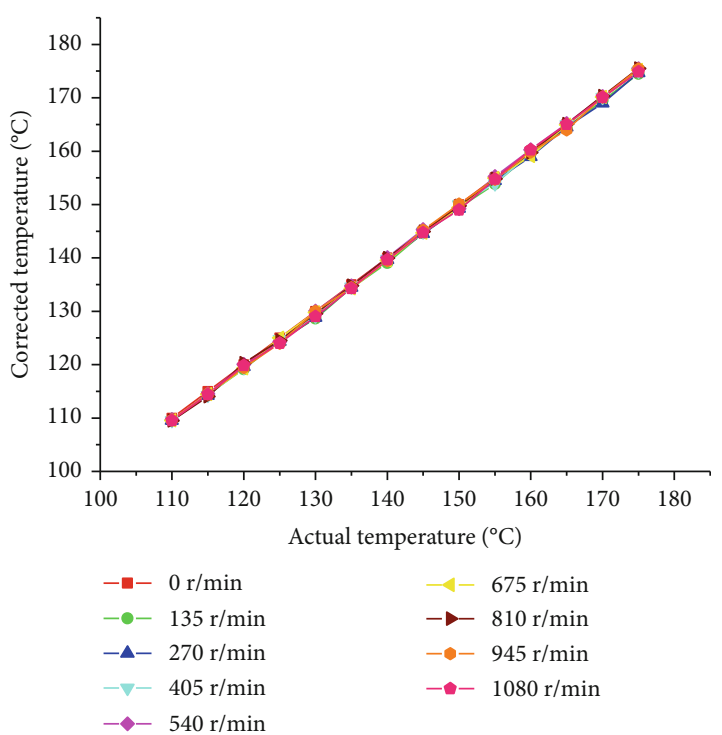

FIGURE 7: Temperature distribution curve after the correction of the compensation algorithm.

ensured that the incident angle of the infrared temperature sensor was always $0^{\circ} \mathrm{C}$. The measurement results are shown in Figure 3, where Figures 3(a)-3(f) correspond to temperature measurements under different rotation speeds at fixed temperatures of $120^{\circ} \mathrm{C}, 130^{\circ} \mathrm{C}, 140^{\circ} \mathrm{C}, 150^{\circ} \mathrm{C}, 160^{\circ} \mathrm{C}$, and $170^{\circ} \mathrm{C}$, respectively.

The green straight line in the figure corresponds to the actual temperature of the bar. The blue curve corresponds to the measurement result of the infrared temperature sensor, and the cyan curve is the error. From Figure 3, it can be found that when the surface temperature of the bar is $120^{\circ} \mathrm{C}, 130^{\circ} \mathrm{C}, 140^{\circ} \mathrm{C}, 150^{\circ} \mathrm{C}, 160^{\circ} \mathrm{C}$, or $170^{\circ} \mathrm{C}$, the temperature data of the bar sample measured by the infrared temperature sensor are different under eight rotation speeds. The measured temperature is lower than the actual temperature. The measurements decrease with increasing rotation speed, and the error increases. The analysis shows that when using infrared temperature sensors to measure the temperature of rotating moving bars, the measurement results always deviate from the true value, and the degree of downward deviation gradually increases with increasing rotation speed. As a result, it is significant to study the error compensation of infrared temperature sensors when measuring the surface temperatures of moving objects.

\section{Infrared Measurement Compensation Algorithms at Different Speeds}

Through the study of the Stefan-Boltzmann law, it is found that the total energy radiated from a unit area of an object's surface in a unit of time is proportional to the fourth power of the thermodynamic temperature of the object. Hence, the temperature change in our experimental results is mainly due to the difference in the amount of radiation received by the infrared temperature sensor. Therefore, the regular change of the infrared measurement results with the rotation speed of the object indicates that the amount of infrared radiation from the object has a certain functional relationship with the object's rotation speed.

Considering the Stefan-Boltzmann law, suppose a variable $m$ is to be measured which is a function of the speed $v$ of the object to be measured. We can define a compensatory factor related to speed. Hence, a new Stefan-Boltzmann formula on the rotational speed can be obtained:

$$
M_{v}=m(v) \cdot \varepsilon(T) \sigma T^{4},
$$

where $M_{v}$ is the amount of radiation from the measured object at speed $v$. In this experiment, the infrared wavelength received by the infrared temperature sensor is $1.6 \mu \mathrm{m}$ and can be considered a constant, so formula (5) can be transformed to

$$
\begin{gathered}
M_{v}=m(v) \cdot k T^{4}, \\
M_{v}=k \cdot\left(m_{T}(v) \cdot T\right)^{4} .
\end{gathered}
$$

$k$ in formula (6) is a constant representing a specific value under specific conditions, determined by $\varepsilon(T)$ and $\sigma$, $k=\varepsilon(T) \bullet \sigma$. Formula (7) can be obtained by combining the function related to the temperature change, where $m_{T}(v)$ is the coefficient of temperature and speed $v$. The change in the quantity of radiation is reflected by the temperature. The temperature value measured by the infrared temperature sensor at different rotating speeds $T_{v}$ is

$$
T_{v}=m_{T}(v) \cdot T .
$$

As shown in Figure 3, the temperature value measured by the infrared temperature sensor changes with the rotation speed of the measured object. By analyzing the measurement results of different rotation speeds at the same temperature, it can be proved that $m_{T}(v)$ exists and preliminary verification of $m_{T}(v)$. To further reveal the variation law of $m_{T}(v)$ , continuous and real-time measurements of continuously varying temperatures were made, as shown in Figure 4.

In Figure 4, nine curves of different colors correspond to the measurement results of nine speeds: $0,135 \mathrm{r} / \mathrm{min}$, $270 \mathrm{r} / \mathrm{min}, \quad 405 \mathrm{r} / \mathrm{min}, 540 \mathrm{r} / \mathrm{min}, 675 \mathrm{r} / \mathrm{min}, 810 \mathrm{r} / \mathrm{min}$, $945 \mathrm{r} / \mathrm{min}$, and $1080 \mathrm{r} / \mathrm{min}$. The abscissa corresponds to the real temperature of the object being measured, and the ordinate corresponds to the actual temperature measured by the infrared temperature sensor. The red curve (curve 1) is the reference curve as well as the standard temperature curve when the speed of the measured object is zero. The other curves are below the red curve; hence, it can be known that the value of the coefficient $m_{T}(v)$ is less than 1 . Comparing the relative position of the reference curve with the other eight curves, it is found that the temperature curve gradually shifts downward as the speed increases, which shows that the actual measured value constantly decreases as the rotation speed increases, and the error gradually increases. The absolute error distribution is shown in Figure 5.

Observing Figure 5, it can be found that the absolute error increases with the rotation speed, and the data 
basically obeys this law under different conditions. However, there are exceptions, which are found to be caused by the following factors:

(1) Light conditions and light changes in the test environment during the experiment

(2) Slippage of the surface thermocouple during the measurement of the experimental sample, causing the thermal equilibrium to be broken

(3) Different response times of the thermocouple and infrared temperature sensor to temperature change, resulting in different measurement results

Considering the accuracy requirements of the engineering tests, the method of constructing a compensation formula is chosen to reduce the measurement error. By observing the data distribution in Figure 4, a linear relationship can be found between the infrared temperature measurement results under different rotation speeds, so the compensation algorithm can be fitted by the least square method.

According to the experimental data in Figures 4 and 5, the compensation formulas of infrared temperature measurement results under different rotation speeds are fitted.

$$
\begin{gathered}
0 \mathrm{r} / \mathrm{min}: T_{v}=0.9998 T-0.1734, \\
135 \mathrm{r} / \mathrm{min}: T_{v}=1.0007 T-0.5859, \\
270 \mathrm{r} / \mathrm{min}: T_{v}=1.0010 T-0.2269, \\
405 \mathrm{r} / \mathrm{min}: T_{v}=1.0040 T+0.2623, \\
540 \mathrm{r} / \mathrm{min}: T_{v}=1.0054 T+0.9663, \\
675 \mathrm{r} / \mathrm{min}: T_{v}=1.0071 T+1.2123, \\
810 \mathrm{r} / \mathrm{min}: T_{v}=1.0093 T+2.0371, \\
945 \mathrm{r} / \mathrm{min}: T_{v}=1.0116 T+2.8597, \\
1080 \mathrm{r} / \mathrm{min}: T_{v}=1.0144 T+3.6762 .
\end{gathered}
$$

Through the analysis of formula (9), it can be found that the primary coefficient value of each compensation formula is close to 1 , so a general compensation formula for speed can be constructed.

$$
T_{b}=T_{v}+\varphi_{T}(v)
$$

where $T_{b}$ is the temperature value after compensation and $\varphi_{T}(v)$ is a functional relationship with the speed of the measured object. By analyzing the compensation formula in formula (9) and making a scatter plot of compensation coefficients, a linear relationship can be found, as shown in Figure 6.

By fitting the curve with the least square method, the general compensation algorithm formula of the infrared temperature sensor measurement results under different speed conditions can be constructed:

$$
T_{b}=T_{v}+(1.46825 E-5) \bullet v+0.3517
$$

In order to verify whether the compensation formula is valid, the actual measurement results of different speeds are compensated for and corrected according to formula (11), and the correction results are shown in Figure 7.

In Figure 7, nine curves of different colors represent the correction curves of the measurement results using the compensation formula under nine speeds. The abscissa is the actual temperature of the measured sample, and the ordinate is the temperature after compensation. By comparing Figures 7 and 4, it can be recognized that the measured data corrected by the compensation algorithm are basically consistent with the actual temperature, which implies that this algorithm can improve the infrared temperature measurement accuracy and stability of the strong reflector at different speeds.

\section{Conclusions}

In this paper, by combining existing laws and experimental data, the principle of infrared temperature measurement is deeply analyzed. The surface temperature of moving objects is measured, and the influence of their rotation speed on infrared temperature measurement results is analyzed. A compensation algorithm based on the Stefan-Boltzmann law is proposed. At the same time, we hope that the algorithm can be extended to different kinds of temperature sensors, such as temperature optical fiber sensors. We draw the following conclusions:

(1) When the rotation speed of the measured object changes, the surface infrared temperature measurement value changes accordingly. The higher the rotation speed, the greater the measurement error, and the measured value deviates from the true value

(2) The infrared temperature measurement compensation formula based on the rotation speed reduces the influence of rotation speed changes on the infrared measurement accuracy and improves the accuracy of the surface temperature measurement of moving objects

\section{Data Availability}

The data used to support the findings of this study are included within the article.

\section{Conflicts of Interest}

The authors declared that they have no conflicts of interest to this work. We declare that we do not have any commercial or associative interest that represents a conflict of interest in connection with the work submitted. 


\section{Acknowledgments}

The authors would like to thank the Institute of Precision Engineering and the Precision and Special Machining Center of the School of Mechanics, Henan Polytechnic University, for their support.

\section{References}

[1] J. Manara, M. Zipf, T. Stark et al., "Long wavelength infrared radiation thermometry for non-contact temperature measurements in gas turbines," Infrared Physics \& Technology., vol. 80, pp. 120-130, 2017.

[2] E. A. Berksoy, O. Bag, S. Yazici, and T. Celik, "Use of noncontact infrared thermography to measure temperature in children in a triage room," Medicine, vol. 97, no. 5, p. e9737, 2018.

[3] E. Lucchi, "Applications of the infrared thermography in the energy audit of buildings: a review," Renewable and Sustainable Energy Reviews., vol. 82, pp. 3077-3090, 2018.

[4] M. Tian and L. Xiong, "Analysis of infrared temperature measurement principle, error and its solution," Instrument Standardization \& Metrology., vol. 2, pp. 40-42, 2016.

[5] Y. Wang, X. Dong, W. Liu, M. Huang, X. Li, and H. Cui, "Research on developments of high temperature testing technology for aero-engine," Measurement \& Control Technology., vol. 36, no. 9, pp. 1-6, 2017.

[6] P. Liao and J. Zhang, "Research on influence factors for measuring and method of correction in infrared thermometer," Infrared Technology., vol. 39, pp. 173-177, 2017.

[7] R. Usamentiaga, D. F. García, and V. M. Passaro, "Infrared thermography sensor for temperature and speed measurement of moving material," Sensors, vol. 17, no. 5, p. 1157, 2017.

[8] S. Masoudi, M. A. Gholami, I. M. Janghorban, and A. Vafadar, "Infrared temperature measurement and increasing infrared measurement accuracy in the context of machining process," Advances in Production Engineering \& Management., vol. 12, no. 4, pp. 353-362, 2017.

[9] Y. Zhang, Y. Chen, X. Fu, and C. Luo, "The research on the effect of atmospheric transmittance for the measuring accuracy of infrared thermal imager," Infrared Physics \& Technology., vol. 77, pp. 375-381, 2016.

[10] W. Chen, Y. Hu, X. Li, and Y. Hong, "Temperature error analysis and compensation of revolving body measuring machine," Nanotechnology and Precision Engineering., vol. 13, pp. 22-27, 2015.

[11] C. Xu, C. Piao, L. Wang, Z. Wang, and J. Tian, “The engine low pressure turbine blade temperature distribution measurement based on the infrared pyrometer technology," Aeroengine., vol. 44, pp. 75-78, 2018.

[12] Y. Zhang, Y. Chen, X. Fu, and C. Luo, "A method for reducing the influence of measuring distance on infrared thermal imager temperature measurement accuracy," Applied Thermal Engineering., vol. 100, pp. 1095-1101, 2016.

[13] Q. Yang, Z. Wei, S. Hu et al., "Application of infrared temperature-measuring technique in temperature monitoring of converter valve," Laser \& Infrared., vol. 48, pp. 73-78, 2018.

[14] D. Fu, Y. Shi, T. Yang, and F. Chen, "Calibration method of infrared measurement of surface temperature for heating furnace tube," Laser \& Infrared., vol. 46, pp. 1486-1490, 2016.

[15] C. Liu, H. Wu, D. Xiong, L. Zhang, and M. Dong, "Nonlinear calibration method of detector in thermal radiation tempera- ture measurement system," Chinese Journal of Quantum Electronics., vol. 34, pp. 227-230, 2017.

[16] F. Meng, Y. Gao, C. Ma, and W. Wang, "Infrared temperature measurement model for molten metal based on thermal radiation theory," Infrared Technology., vol. 39, pp. 766-771, 2017. 